\title{
Microbiological characteristics of dermatological patients with ulcers of the lower extremities
}

\author{
Useliene $\mathrm{J}^{1,2}$, Gyliene $\mathrm{I}^{1,2}$, Lauraitis $\mathrm{J}^{2}$, Grigaitiene $\mathrm{J}^{1,2}$, Bylaite-Bucinskiene $\mathrm{M}^{1,2}$. \\ ${ }^{1}$ Centre of Dermatovenereology, Vilnius University Hospital Santariskiu Clinics \\ 2Vilnius University
}

The aim of the study

Treatment of ulcers of the lower extremities is challenging and complicated by many aspects. One of them is a wide variation of pathogenic bacteria, course of the disease.

The aim of this study was to determine and evaluate microbiological pattern

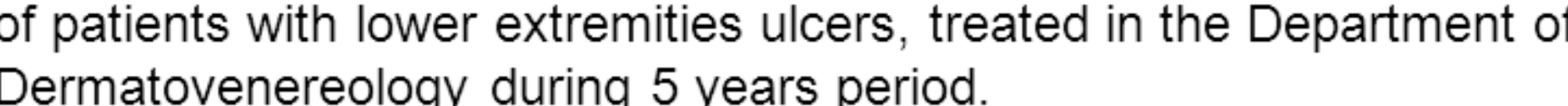
toloring 5 years period.
Materials and Methods

A retrospective 5 -year period analysis of total 429 patients clinical and medical documentation data was performed. mean (SEM).

A value of $p<0.05$ was considered statistically significant
Data of patients demographics, anamnesis of the disease, physical,

(SPS Inc.

Coherence between repeated hospitalisation and bacterial profile of the ulcers

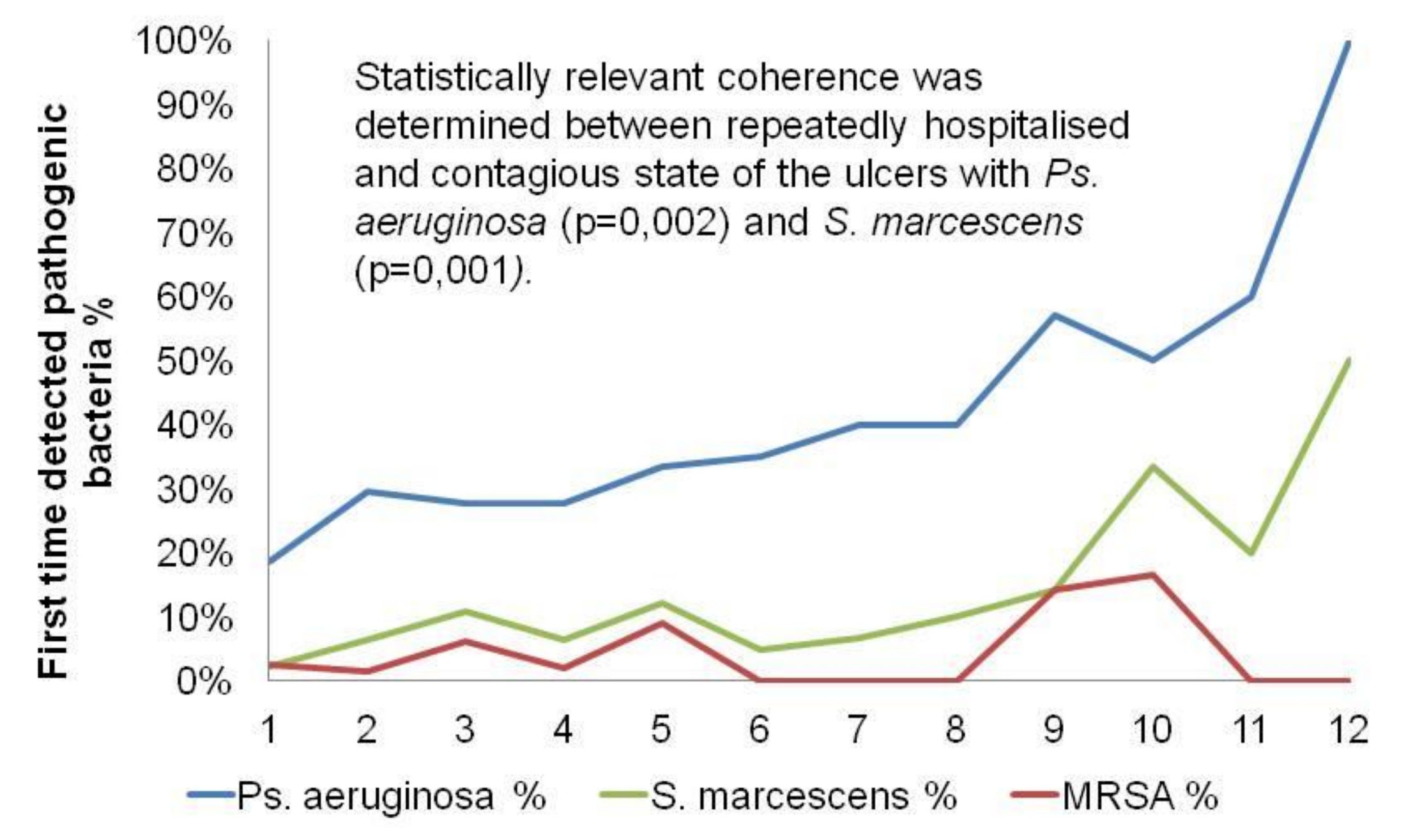

Bacteria distribution according to patients age

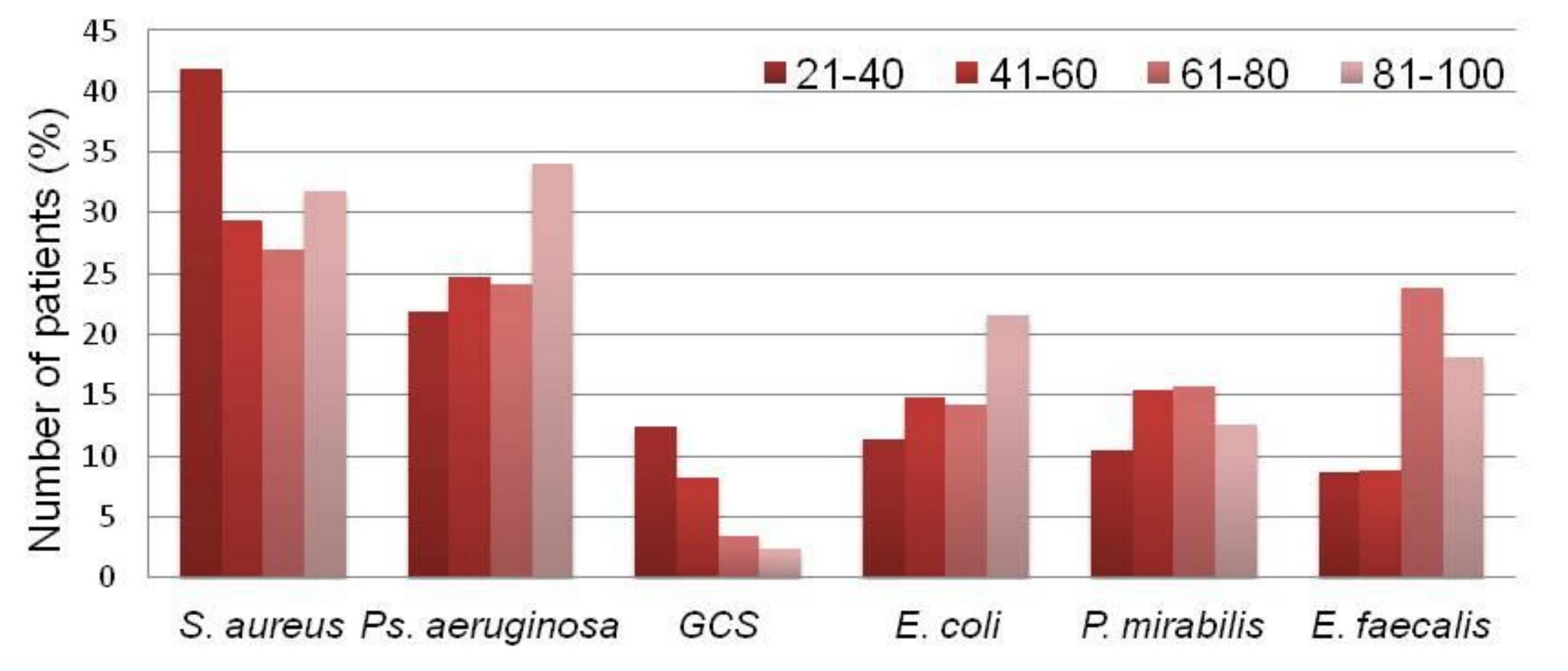

St. $\beta$-haemolyticus $C$ gr. (GCS) is inherent to younger patients group $21-40(p=0,002)$ E. faecalis is inherent to elderly patients groups: $61-80,80-100$ years old $(p<0,001)$.

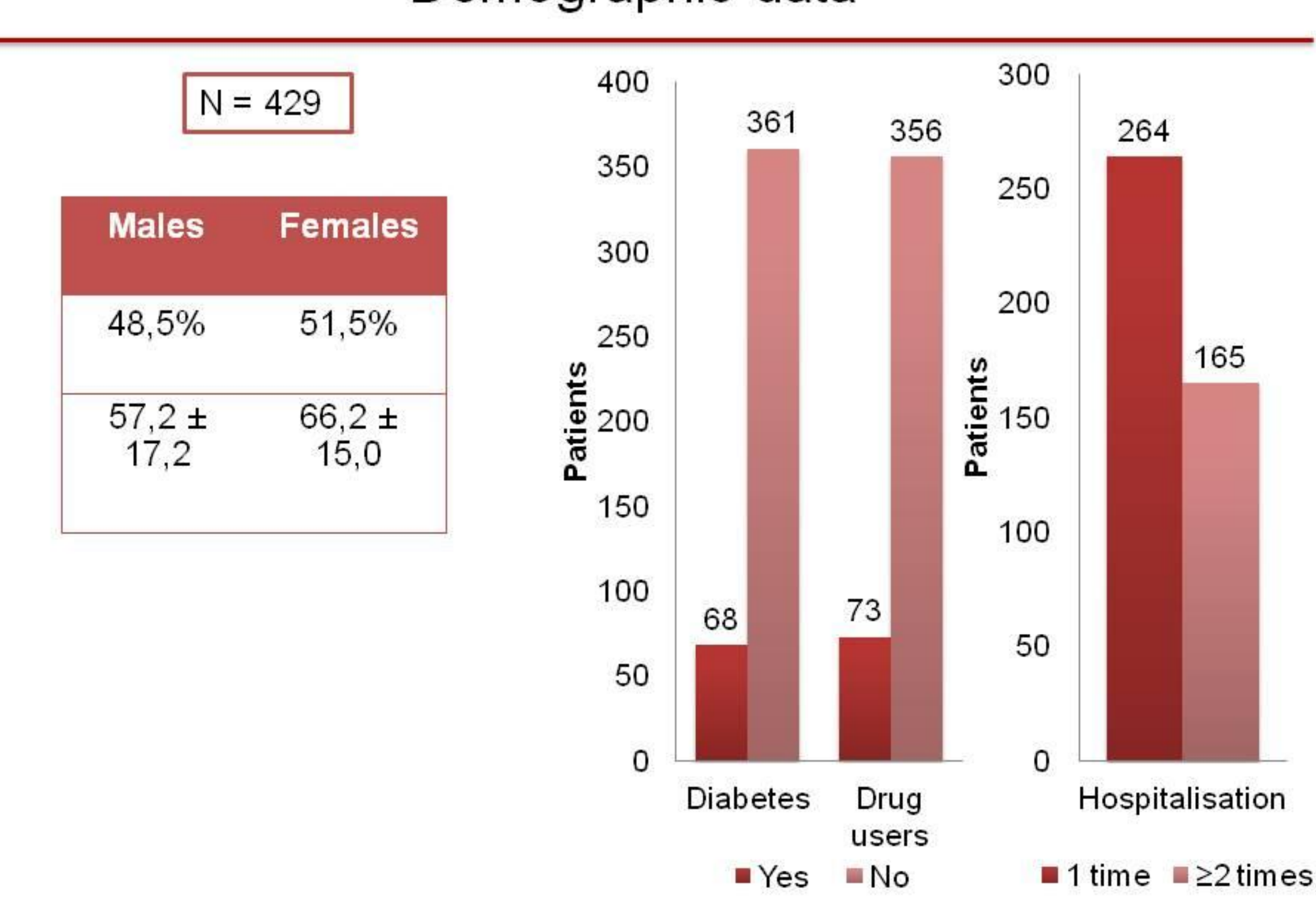

Most common bacteria cultivated from swab tests

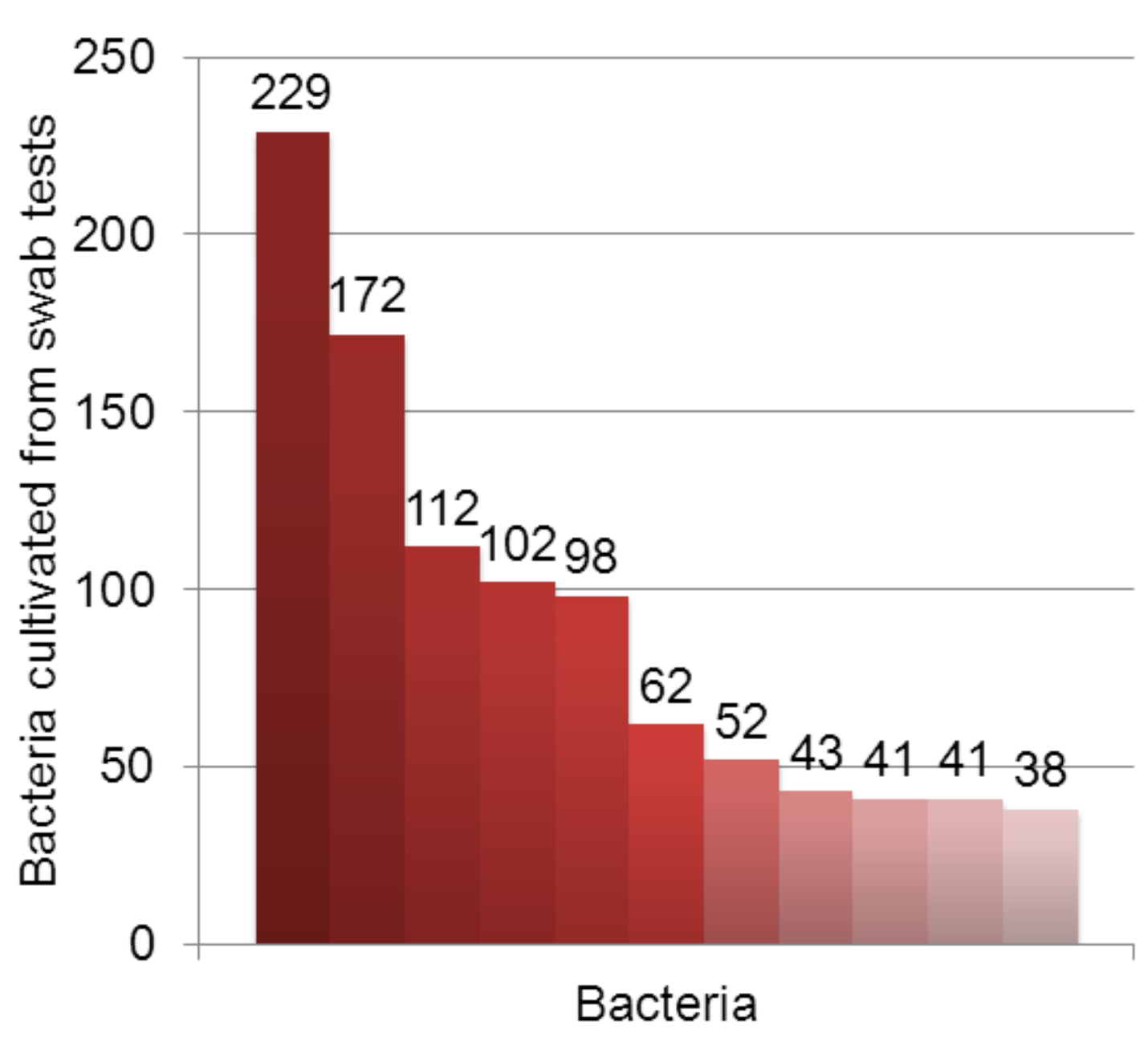

S. aureus

- Ps. aeruginosa

- E. coli

- P. mirabilis
- K. oxytoca

- M. morganii

- $S$. B haemolyticus $B$ gr.

S. B haemolyticus A gr.

S. marcescens
Bacterial profile specifics of leg ulcers in patients with diabetes

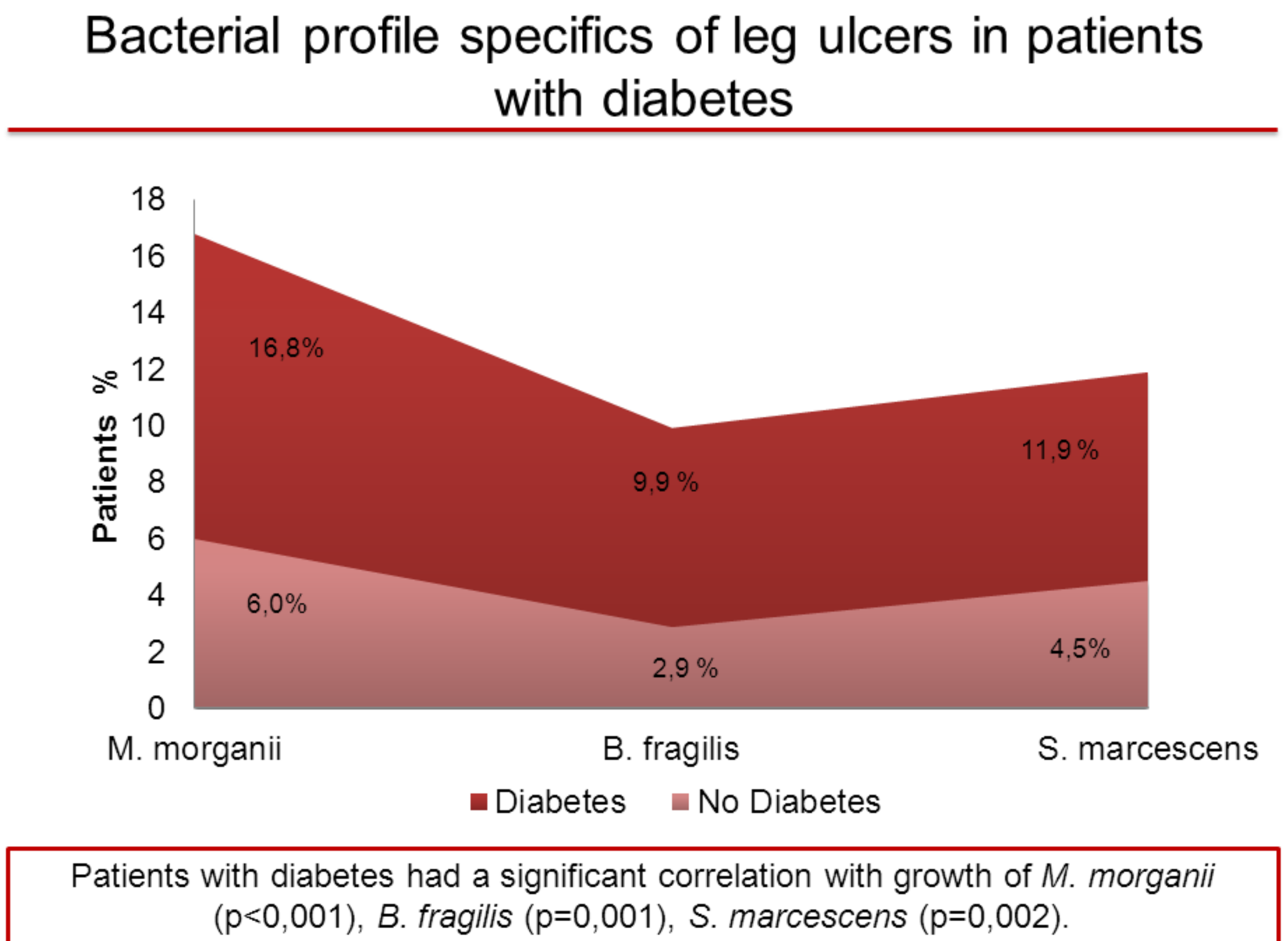

Bacterial profile specifics of leg ulcers in drug addicted patients

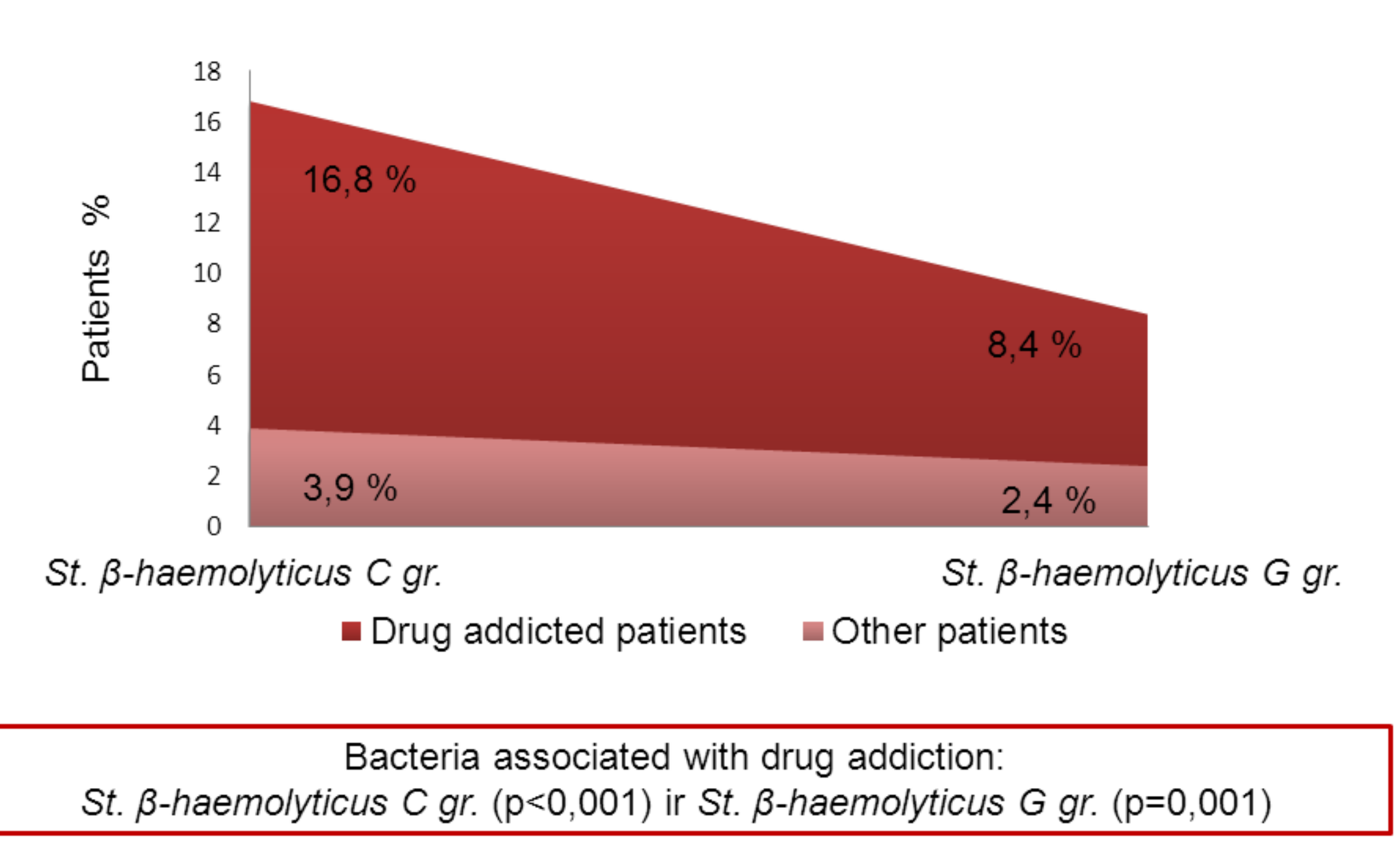

Tendency of leg ulcer treatment in Vilnius University Santaros Clinics Dermatovenereology department

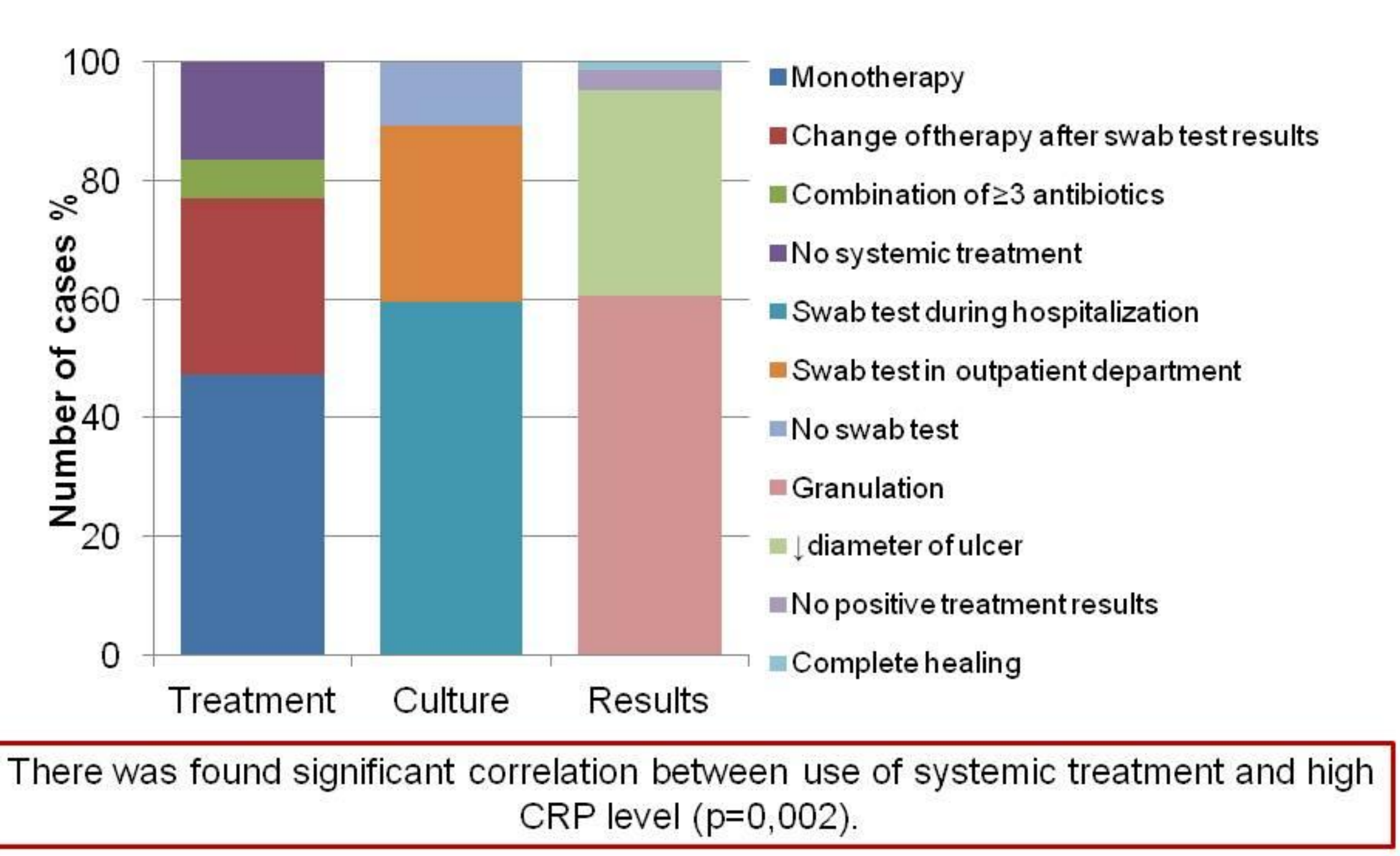

Mostly used and at least effective antibiotics

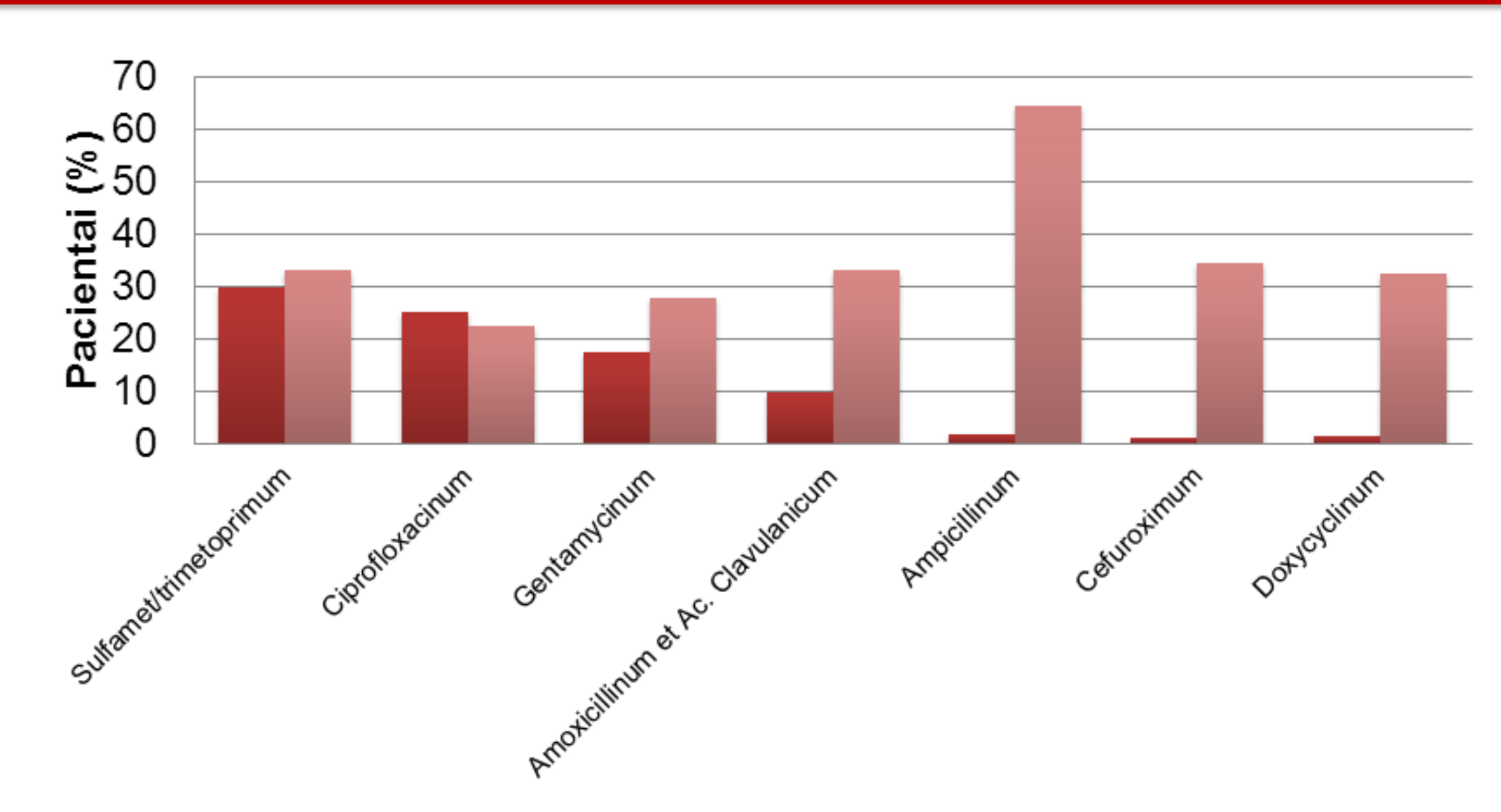

- Used antibiotics $\quad$ Bacteria resistance

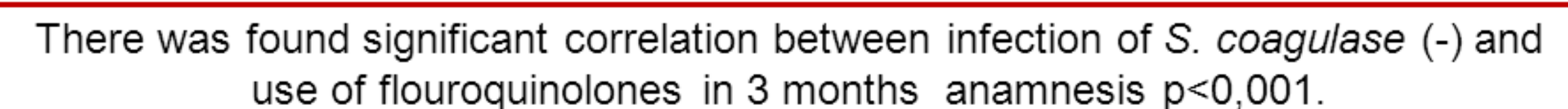

Tendency of bacteria resistance to antibiotics during $2010-2015$ years period

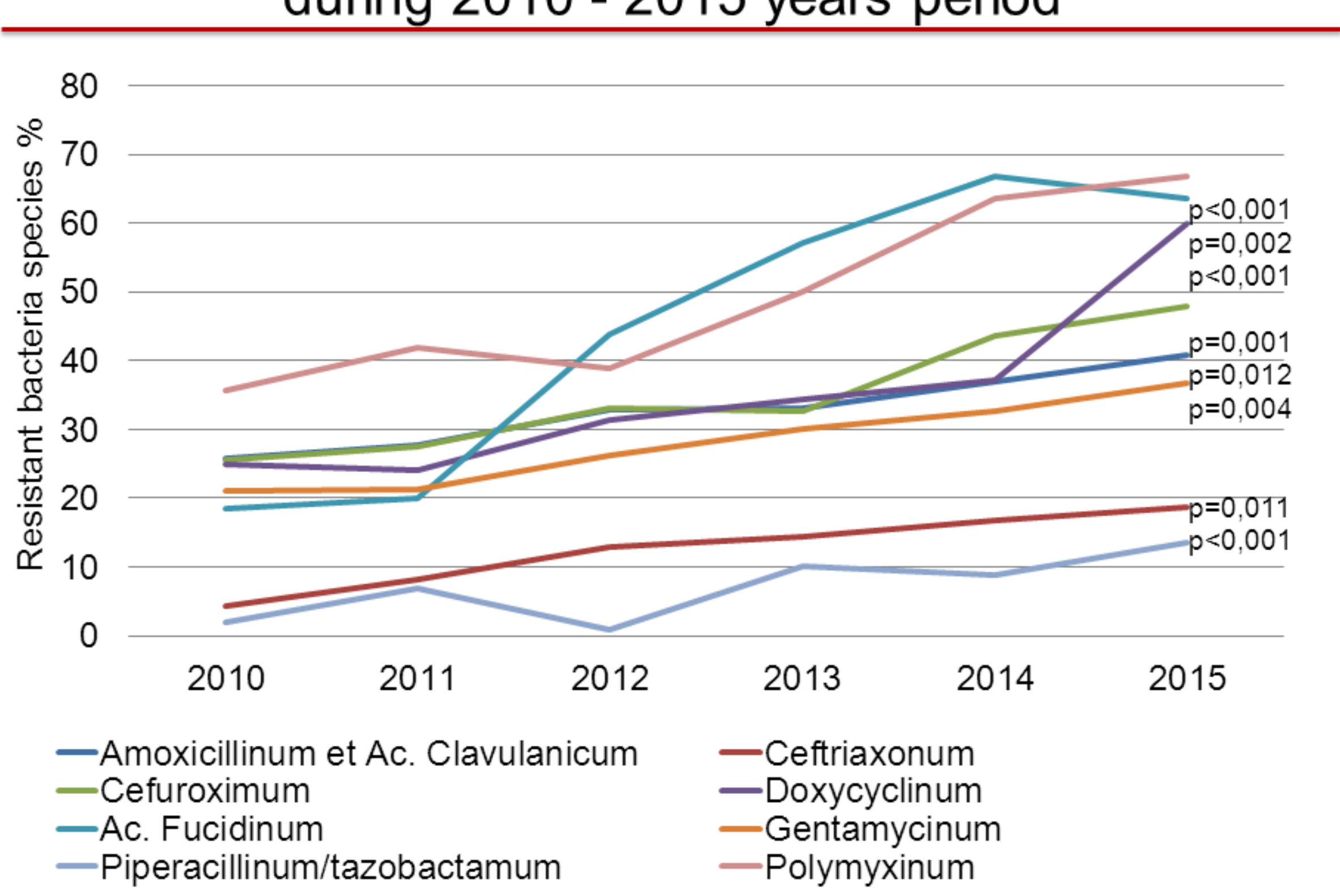

Conclusions

Ulcers of the lower extremities remains a chronic health care problem which is often complicated by secondary infectious disease.

From culture of leg ulcers most frequently growing bacteria are: St. aureus, Ps. aeruginosa and E. faecalis.

A significant correlation between repeatedly treated patients and detection

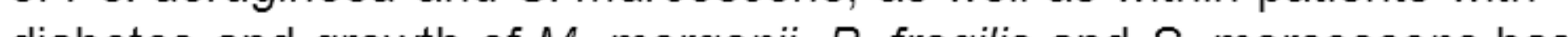

Abundant usage of antibiotics in treatment of lower extremities ulcers increases rise and spread-out of resistant pathogenic microorganism
Thus, the antibacterial treatment should be selected with caution. 